\title{
Getting stuck in social isolation: Solitude inertia and depressive symptoms
}

Timon Elmer ${ }^{1}$, Nicole Geschwind ${ }^{2}$, Frenk Peeters ${ }^{2}$, Marieke Wichers ${ }^{3}$, \& Laura Bringmann ${ }^{3,4}$

${ }^{1}$ Chair of Social Networks, Department of Humanities, Social and Political Sciences, ETH Zürich, Switzerland

${ }^{2}$ Maastricht University, Faculty of Psychology and Neuroscience, Department of Clinical Psychological Science, Maastricht, The Netherlands

${ }^{3}$ University of Groningen, University Medical Center Groningen, Department of Psychiatry, Interdisciplinary Center Psychopathology and Emotion regulation (ICPE), Groningen, The Netherlands

${ }^{4}$ University of Groningen, Faculty of Social and Behavioural Sciences, Department of Psychometrics and Statistics, Groningen, The Netherlands

In press in the Journal of Abnormal Psychology

Author Note. The data collection was funded by the Dutch Organisation for Scientific Research (NWO, VENI Grant Nr. 916.76.147 for M. Wichers. The data collection and intended uses were approved by the Medical Ethics Committee of Maastricht University Medical Centre (trial number NTR1084, Netherlands Trial Register). Correspondence concerning this article should be addressed to Timon Elmer, Chair of Social Networks, Department of Humanities, Social and Political Sciences, Weinbergstrasse 109, 8092 Zürich, Switzerland. Email:

timon.elmer[at]gess.ethz.ch

(C) 2020, American Psychological Association. This paper is not the copy of record and may not exactly replicate the final, authoritative version of the article. Please do not copy or cite without authors' permission. The final article will be available, upon publication, via its DOI:

10.1037/abn0000588 


\begin{abstract}
Social isolation and depression are tightly linked and can reinforce each other in a vicious cycle. Yet, the antecedents of this complex cycle are not well understood. To date, little is known about how the dynamics of social isolation in daily life (i.e., solitude) play a role in this cycle. To investigate these complex dynamics, we introduce the concept of solitude inertia, which captures individuals' tendencies to remain in social states of solitude. We argue that, although short-term solitude can have both positive and negative effects on individuals' depressive symptoms, prolonged states of solitude (i.e., high solitude inertia) are detrimental. At the same time, individuals with depression might be more vulnerable to 'get stuck' in solitude. In this study, we tease apart the bidirectional relationship between solitude inertia and depressive symptoms. We use data from the MindMaastricht study in which 129 individuals with residual depressive symptoms participated in two experience sampling assessments phases (T1 and T2) that were eight weeks apart $\left(\mathrm{N}_{\mathrm{obs}}=11,558\right)$. Using logistic multilevel models, we find that higher levels of depressive symptoms are related to higher solitude inertia. We further show that depressive symptoms at T1 are not predictive of solitude inertia at T2. However, solitude inertia at T1 was predictive of depressive symptoms eight weeks later (T2) in a linear regression analysis. This study introduces and highlights the role of solitude inertia as a potential intervention target in social isolation and depression dynamics.
\end{abstract}

Keywords: Solitude, Social Isolation, Solitude Inertia, Depression, Dynamics, Social Network 


\section{General Scientific Summary}

This study introduces the concept of solitude inertia, which captures individuals' tendencies to remain in solitude over time, and examines its bidirectional associations with depressive symptoms. The findings of this study suggest that depressive symptoms are not predictive of solitude inertia but that solitude inertia is positively associated with depressive symptoms eight weeks later. Thus, solitude inertia might be a promising intervention target for individuals suffering from depressive symptoms. 


\section{Introduction}

Humans are fundamentally motivated to interact with others (Baumeister \& Leary, 1995). Socially isolated individuals generally report lower levels of mental health (Kawachi \& Berkman, 2001). More specifically, individuals with fewer supportive social relationships are more likely to suffer from depression (e.g., Cohen \& Ashby, 1985; Santini, Koyanagi, Tyrovolas, Mason, \& Maria, 2015). The relationship between social isolation and depression is bidirectional: while social isolation might lead individuals to develop more depressive symptoms, depressive symptoms might also affect the extent to which individuals exhibit social isolation (Elmer, Boda, \& Stadtfeld, 2017; Rottenberg \& Gotlib, 2008). These reinforcing patterns potentially lead to a vicious cycle of social isolation and depression. However, the antecedents of this vicious cycle are not yet well understood.

Social isolation is a complex macro-level phenomenon that is best investigated in terms of a dynamic flow of daily social experiences on the micro-level (Chappell \& Badger, 1989; Gable \& Reis, 1999; Wichers, 2014). On this micro-analytic level, social isolation manifests in spending time alone. To date, however, it remains unclear how the micro-level dynamics of solitude in daily life contribute to the vicious cycle of macro-level social isolation and depression. This study aims at filling this gap.

On the micro-level, being alone is not necessarily bad: Although being alone can have negative effects on mental health, there are also positive ones (Coplan, Zelenski, \& Bowker, 2017). For instance, having some alone time during the course of a day is essential for an individual's social balance and recovery (Larson, 1990; Long \& Averill, 2003). This dynamic self-regulation suggests that not just the sheer number of social interactions is important for depression but also the patterns in which social interactions and solitude occur. In this article, we argue that contradictory views on the merits of solitude appear because the dynamics in which solitude occurs are not considered.

To capture these solitude dynamics at the microlevel, we introduce the concept of social inertia, which describes an individual's tendency to remain in social states over time ${ }^{1}$. In the context of solitude, solitude inertia captures the tendency to stay in prolonged states of being

\footnotetext{
${ }^{1}$ In Sociology, the concept of social inertia refers to the resistance to change in stable relationships in society or social groups (e.g., social class; Bourdieu, 1985). The opposite of social inertia is social change. In this article, we are expanding the meaning to the psychological sciences.
} 
alone. In other words, individuals with low solitude inertia tend to switch more frequently between states of alone and social interactions. Figure 1 (left side) illustrates the concept of solitude inertia on an example of high and low solitude inertia, with the time spent in social interactions being identical in both cases.

While the relations between social isolation and depression have received much scholarly attention, little is known about how the micro-dynamics of solitude inertia manifest themselves as consequences and/or antecedents of depression. The concept of solitude inertia is novel and its relations to individuals' depressive symptoms are not yet known. What are the processes that potentially explain why solitude inertia and depressive symptoms are linked? In the following, we will review the literature on, first, how depressive symptoms might affect solitude inertia and, second, how solitude inertia might affect the development of depressive symptoms.

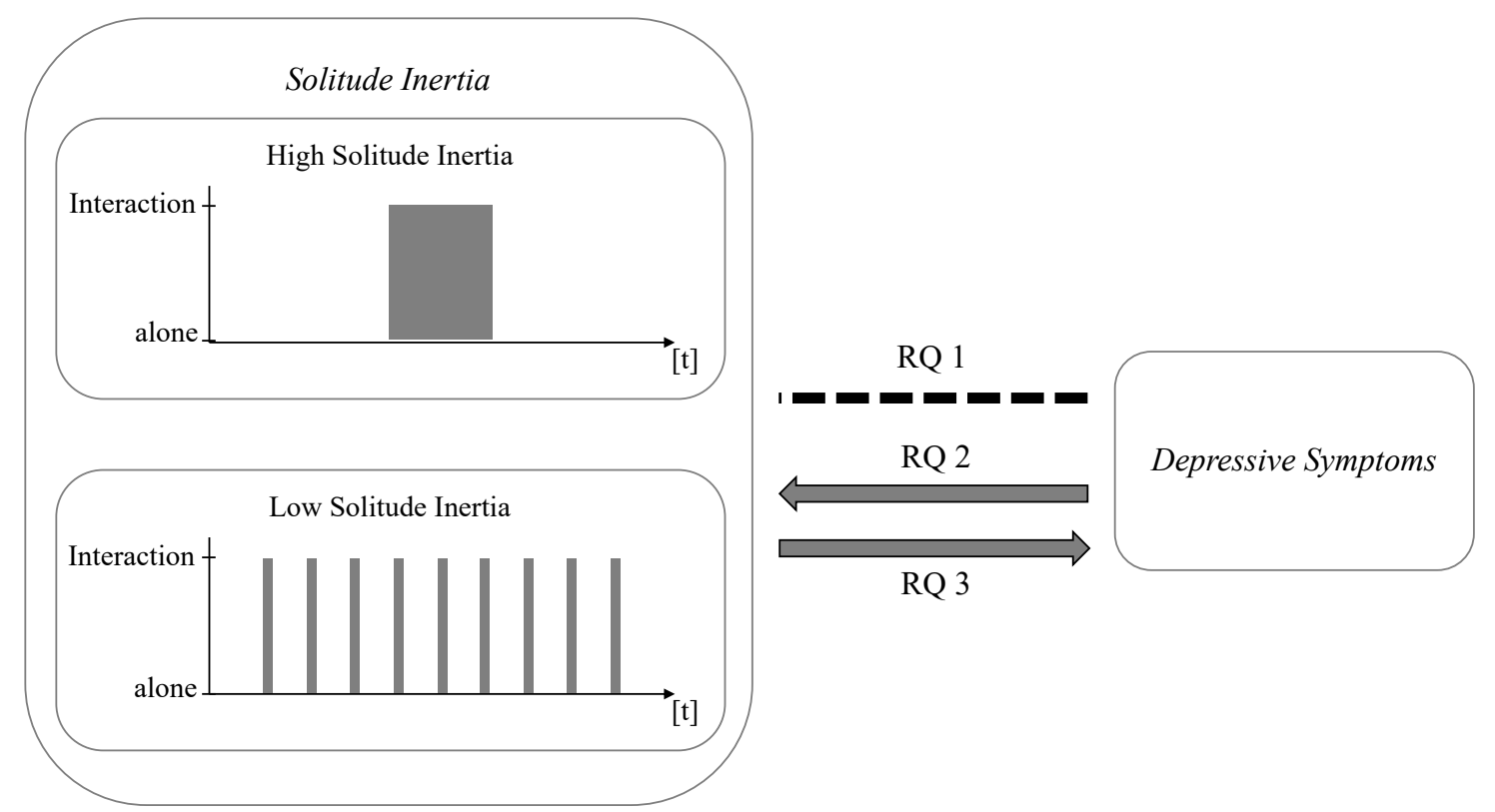

Figure 1. Research Questions (RQ) and illustration of high and low solitude inertia (note that the total amount of time spent alone is equal). RQ 1 examines concurrent associations between solitude inertia and depressive symptoms, while RQ 2 and 3 examine longitudinal relationships.

\section{The effect of depressive symptoms on solitude inertia}

Social behavior of more severely depressed individuals is generally more dysfunctional than the behavior of less severely depressed individuals (Rottenberg \& Gotlib, 2008). In particular, social anhedonia, or the "disinterest in social contact and diminished pleasure derived from social contact" (Kwapil, Silvia, \& Barrantes-Vidal, 2013, p. 371), is associated with depressive 
symptoms (e.g., Atherton, Nevels, \& Moore, 2015). As a result, individuals with more depressive symptoms are less motivated to seek social contact in general, but most importantly when they are already alone (Silvia \& Kwapil, 2011). Less depressed individuals, at the same time, might be motivated to seek social contact after being alone. Once an individual with high depressive symptoms is alone, it gets harder for that person to engage in social interactions again. This way, the person can 'get stuck' in social isolation. Hence, higher depressive symptoms should predict more pronounced solitude inertia, both contemporaneously and over a longer period of time.

\section{The effect of solitude inertia on the development of depressive symptoms}

We further investigate how the micro-level dynamics of social life aggregate to affect depressive symptoms. This micro-macro link between daily (social) experiences and mental health has been put forward by numerous theorists (e.g., Fredrickson \& Joiner, 2002; Wichers, 2014). In particular, it is argued that "reinforcing loops between momentary states may alter the course of mental health towards either a more or less healthy state" (Wichers, 2014, p. 1). Might solitude inertia be part of such a reinforcing loop?

Similarly to a 'need to belong' (Baumeister \& Leary, 1995) people also have a 'need for solitude' (Long \& Averill, 2003). Whether or not solitude has a positive effect on an individual's well-being and mental health might depend on the dosage and appraisal of company (for a review see Coplan et al., 2017). Scholars have drawn the analogy to sunshine - from time to time some skin exposure is healthy but too much in a short period of time can cause sunburn (Coplan et al. 2017). In this vein, Larson (1990) reported that in adolescent populations a moderate amount of time in solitude is associated with lower levels of psychological distress relative to those adolescents who spent either less or more time alone. Nevertheless, existing studies only investigate the total amount of solitude and not the patterns in which solitude occurs. In line with the sunshine analogy, we suggest that not necessarily the total amount of solitude (i.e., social isolation) but the dynamic patterns of solitude should affect depressive symptoms. So how do the dynamic patterns of solitude affect depressive symptoms?

Prolonged states of solitude (i.e., solitude inertia) facilitate a number of symptoms of depression - in particular rumination (Evans, Marsh, \& Owens, 2007). Social interaction can help to get out of ruminating states and regulate emotions (e.g., see Nolen-Hoeksema \& Davis, 1999). When social interactions are absent for longer periods of time, excessive rumination can 
lead to the development of other depressive symptoms (Nolen-Hoeksema, 2000). Similarly, it has been argued that social contact helps to maintain a sense of reality and sanity (Baumeister \& Leary, 1995). Thus, we expect individuals who exhibit more solitude inertia to have developed more depressive symptoms later on.

\section{The present study}

To test these predictions, in a first step we explore whether solitude inertia and depressive symptoms are positively associated with each other (research question [RQ]1). In a second step, we aim at disentangling the directionality of this association through longitudinal comparisons: Are depressive symptoms at baseline predictive of solitude inertia at postmeasurement (RQ2)? And is solitude inertia at baseline predictive of depressive symptoms at postmeasurement (RQ3)? 


\section{Methods}

\section{Participants}

The data for our analyses stem from the MindMaastricht study (Geschwind, Peeters, Drukker, Van Os, \& Wichers, 2011) in which 129 adults with residual symptomology of a major depressive disorder participated. The majority of participants was female $(\mathrm{N}=98 ; 76 \%)$ and their mean age was $43.79(\mathrm{SD}=9.60)$ years. $95 \%$ of the participants were born in the Netherlands, the remaining 5\% were from either Germany, Belgium, or Norway. About 13\% of the participants regularly had psychotherapeutic counseling and 35\% used antidepressant medication. Only participants that did not have planned changes in their psychological or pharmacological treatment were included in this study.

\section{Procedure}

Participants were recruited from outpatient mental health care facilities as well as through advertisements in the Netherlands. Eligible participants (see Geschwind et al., 2011) were invited to the baseline assessment (T1) which consisted of a six-day experience sampling assessment, followed by a diagnostic interview in which the extent to which participants experience depressive symptoms was determined (see the Measures section). After the baseline assessment (T1), participants were randomly assigned either to an eight-week Mindfulness-Based Cognitive Therapy program ( $\mathrm{N}=63,49 \%$; MBCT; Teasdale et al., 2000) or a waiting list (control group). Thereafter, the post-assessment (T2) was conducted that included another six-day experience sampling assessment and the same diagnostic interview. Figure 2 shows the longitudinal study design of the MindMaastricht study.

In each of the two experience sampling assessments, participants were 'beeped' at (semi)random time points during their daily lives to fill out a short questionnaire assessing their current emotional and social experiences. Each participant received ten such invitations a day for six subsequent days. For further details on the study procedure see Geschwind et al. (2011). 


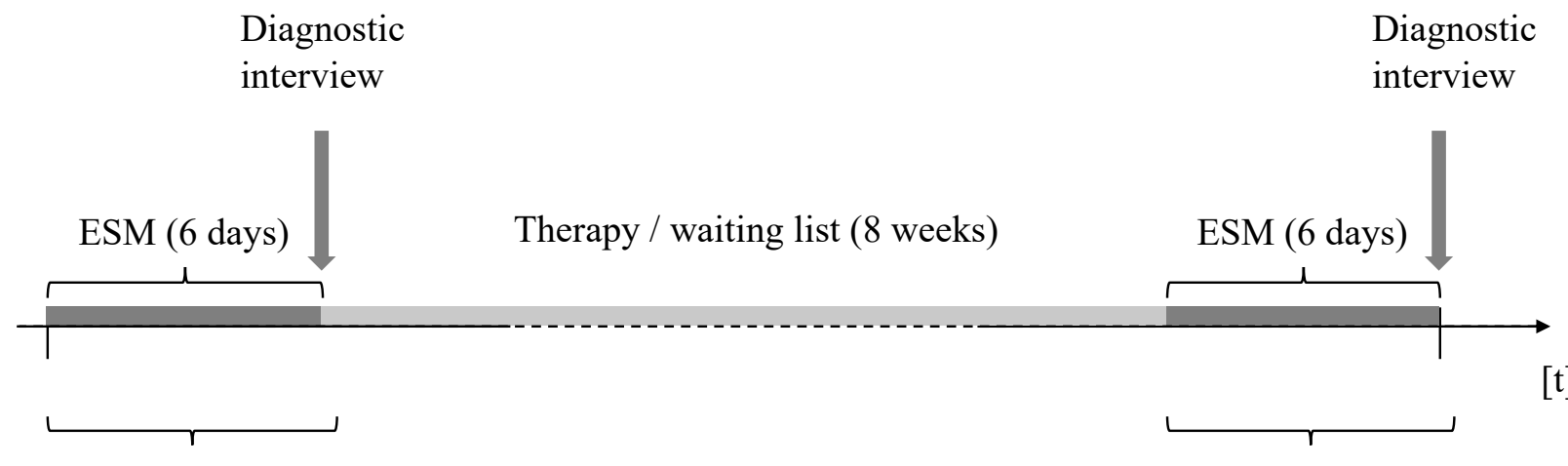

$\mathrm{T} 1$

$\mathrm{T} 2$

Figure 2. Study design of the MindMaastricht study (Geschwind et al., 2011). The ESM period refers to the experience sampling assessment that consisted of ten measures a day for six consecutive days.

\section{Measures}

Being alone. In each experience sampling assessment, participants were asked whether they were alone or in company of others $(0=$ not alone; $1=$ alone $)$.

Solitude inertia. Solitude inertia was operationalized as the autocorrelation between two subsequent measures of being alone. In other words, solitude inertia is captured in the regression coefficient of the previous experience sampling assessment of being alone on the next one. Similar operationalizations using autocorrelations have been used to study emotional inertia (Dejonckheere et al., 2019; Kuppens, Allen, \& Sheeber, 2010; Suls, Green, \& Hillis, 1998). When estimating the autocorrelation with measures of previously being alone, we excluded the observations from the previous evening, i.e., the last measure of the day is not used to predict the first measure of the next day. The reason for this exclusion is that the time interval between these measures is much longer than the time interval between measures during the day, which violates the assumption of equal spacing inherent in our multilevel model (de Haan-Rietdijk, Voelkle, Keijsers, \& Hamaker, 2017).

Social isolation. We operationalized social isolation as the percentage of experience sampling assessments in which a person reported to be alone (i.e., alone ratio). We normalized the total number of alone reports by each participants number of responses, as the number of 
responses can differ between individuals. The following formula shows the calculation of the social isolation / alone ratio variable $x$ for a given individual $i$ :

$$
x_{i}=\frac{\sum_{k}^{n_{i}} x_{i k}}{n_{i}}
$$

Where $n_{i}$ denotes the number of observations of individual $i$. Participants that were always alone would have a score of 0 and participants that were never alone have a score of 1 . When investigating the dynamics of a particular phenomenon it is important to take into account the effects of the mean level (Dejonckheere et al., 2019). In this case, we thus control for the main effect of being alone (i.e., general social isolation) on depressive symptoms when investigating the dynamics of being alone.

Depressive Symptoms. In the diagnostic interview assessments, the 17-item Hamilton Rating Scale for Depression (HDRS; Hamilton, 1960) was administered by trained psychology graduates. The semi-structured interview assesses the degree to with individuals experienced depressive symptoms over the past week. An example item of the HDRS to assess depressed mood (sadness, hopeless, helpless, worthless) is coded as "Absent" (0), "These feeling states indicated only on questioning” (1), “These feeling states spontaneously reported verbally" (2), "Communicates feeling states non-verbally, i.e. through facial expression, posture, voice and tendency to weep" (3), "Patient reports virtually only these feeling states in his/her spontaneous verbal and non-verbal communication.” (4). The HDRS score ranges between zero and 52.

Control variables. Age and gender were assessed together with the first measure of depressive symptoms.

\section{Analytical Strategy}

The analysis is divided according to the three research questions. To examine the concurrent association between solitude inertia and depressive symptoms (RQ1) we conduct an analysis of the "being alone" ESM variable measured during T1 with the depressive symptom measures assessed with a diagnostic interview about the same time period. The directionality between 
depression and solitude inertia is disentangled in a longitudinal matter in RQ2 and RQ3. To investigate RQ2, we examine the effect of T1 depressive symptoms on T2 solitude inertia. To investigate RQ3, we longitudinally assess the effects of T1 solitude inertia on the development of depressive symptoms at T2. As this is a secondary data analysis, no a priori power analyses have been conducted.

The association between solitude inertia and depressive symptoms (RQ1). We estimate timelagged logistic multilevel models, where experience sampling observations (Level 1) are nested within individuals (Level 2). The dependent variable is the state of being alone $(0=$ not alone; $1=$ alone) during the experience sampling assessment in T1. To study solitude inertia, we study the effects of previously being alone on being alone (i.e., the alone autocorrelation). The effect of depressive symptoms measured at the end of period T1 is another independent variable in predicting reports of being alone. Also, we add the interaction of the alone autocorrelation (solitude inertia) and depression to the model. This interaction effect indicates whether more depressed individuals, as hypothesized, are more likely to be alone when they were previously alone. Further, we control for the effects of gender and age on being along. The individual intercept and the alone autocorrelation slope are modeled as random variables.

The effect of depressive symptoms on solitude inertia (RQ2). This analysis again uses timelagged logistic multilevel models. The difference to Part I lies in the cross-lagged variables: Depressive symptoms at T1 are used to predict dynamics of being alone during T2. In other words, we estimate solitude inertia during the second ESM period (T2) while considering how depressed individuals were during the first ESM period (T1 measured once). With the interaction of solitude inertia during T2 and depressive symptoms T1, we can assess if individuals with more depressive symptoms are more likely to exhibit solitude inertia eight weeks later. Aside from an additional control variable for the main effect of the therapy intervention and its interaction with the solitude inertia parameter, the model specification is identical to the one in Part I.

The effect of solitude inertia on depressive symptoms (RQ3). We assess the effect of T1 solitude inertia on depressive symptoms at T2 with linear regression models. Predictors include 
covariates for age, gender, and depressive symptoms at T1 (thus only modeling the change in depressive symptoms between $\mathrm{T} 1$ and T2). Also, we control for the ratio of being alone during T1 (social isolation) and effect of the treatment intervention, as treatment was found to have a positive effect on the reduction of depression symptomatology (Geschwind et al., 2011). For this research question, the most important independent variable is the T1 solitude inertia. RQ3 requires to be tested in a different statistical framework than RQ1 and RQ2 because the dependent variable is now on Level 2 (one T2 depression measure per individual) and not anymore on Level 1 (repeated alone observations nested within individuals). To assess the effect of $\mathrm{T} 1$ solitude inertia on depression at T2, we therefore extracted the (Level 2) individual random slopes of the alone autocorrelation of the time-lagged logistic multilevel model estimated in the analysis for RQ1. These individual slope coefficients are often referred to as best linear unbiased predictors (BLUP) or the empirical Bayes (EB) estimates (Liu, Kuppens, \& Bringmann, 2019; Neubauer, Voelkle, Voss, \& Mertens, 2019). In this context, they represent the estimated effect of the previous alone measurement on the next one (i.e., their autocorrelation), and thus is a measure for an individual's solitude inertia. The extraction of BLUP values and their use as predictors in subsequent analyses has been frequently applied in psychological research to assess the effects of individual dynamics on another variable (e.g., Brose, Schmiedek, Koval, \& Kuppens, 2015; Kuppens et al., 2010). 


\section{Results}

In a first step, we report how solitude inertia dynamics (i.e., alone autocorrelations) during the first experience sampling assessment period (T1) are associated with depressive symptoms (HDRS interviews) reported for the same period (RQ1). In a second step, we report how T1 depressive symptoms predict solitude inertia dynamics eight weeks later at T2 (RQ2). In the third step (RQ3), we investigate how the dynamics of being alone (captured through individual BLUP coefficients at T1) predict depression scores eight weeks later (T2).

\section{Participation Rates}

On average, participants responded to $47.05(\mathrm{SD}=8.03)$ of the 60 experience sampling assessments in $\mathrm{T} 1$ and $46.12(\mathrm{SD}=8.89)$ of 60 in $\mathrm{T} 2$. In total there are 6,070 experience sampling data points for $\mathrm{T} 1$ and 5,488 data points for T2. We selected the datapoints with two subsequent "being alone" measures (T1 = 4,725 observations; T2 = 4,239 observations) and ignored the datapoints for which there was either a missing observation for the current "being along" measure or for the previous measure ( $\mathrm{T} 1=1,345$ observations; $\mathrm{T} 2=1,249$ observations). Of the 129 individuals who started the study in $\mathrm{T} 1,92 \%(\mathrm{~N}=119)$ also participated in $\mathrm{T} 2$. The T2 depression interview was conducted on 123 individuals.

\section{Descriptives}

On average, individuals reported to be alone in $43 \%$ of the experience sampling measures $\left(\mathrm{N}_{\mathrm{alone}}\right.$ $\left.=2,593, \mathrm{~N}_{\text {Total }}=6,070\right)$. Figure 1 in the Supplementary Material shows two example trajectories of social interactions during T1. The mean HDRS depression score at T1 was $10.30(\mathrm{SD}=3.52)$ and $8.44(\mathrm{SD}=4.56)$ at $\mathrm{T} 2$. During the 6 days of $\mathrm{T} 1$, on average participants reported to be alone twice in a row $10.20(\mathrm{SD}=8.06)$ times, thrice in a row $5.72(\mathrm{SD}=6.52)$ times, and $3.36(\mathrm{SD}=$ 5.10) being alone four times in a row. The number of alone sequences during T1 correlated moderately with depression at $\mathrm{T} 1\left(\mathrm{r}_{\text {twice }}(127)=.180, \mathrm{p}_{\text {twice }}=.042 ; \mathrm{r}_{\text {thrice }}(127)=.180, \mathrm{p}_{\text {thrice }}=.041\right.$; $\left.\mathrm{r}_{\text {four }}(127)=.172, \mathrm{p}_{\text {four }}=.052\right)$ and marginally significant with depression at $\mathrm{T} 2\left(\mathrm{r}_{\text {twice }}(121)=.162\right.$, $\mathrm{p}_{\text {twice }}=.074 ; \mathrm{r}_{\text {thrice }}(121)=.174, \mathrm{p}_{\text {thrice }}=.055 ; \mathrm{r}_{\text {four }}(121)=.161, \mathrm{p}_{\text {four }}=.075$. The probability of remaining alone in two consecutive reports during T1 correlated with depression at $\mathrm{T} 1(\mathrm{r}(128)=$ $.22, \mathrm{p}=.011)$ or $\mathrm{T} 2(\mathrm{r}(128)=.19, \mathrm{p}=.027)$. On the other hand, the probability of remaining in a social interaction in two consecutive reports during T1 did not correlate with depression at T1 $(\mathrm{r}(128)=.03, \mathrm{p}=.721)$ or $\mathrm{T} 2(\mathrm{r}(128)=-.01, \mathrm{p}=.903)$. 


\section{The association between solitude inertia and depressive symptoms (RQ1)}

Figure 3 presents the odds ratio estimates of the logistic multilevel model on being alone. Of particular importance for RQ1 are the estimates of depressive symptoms, solitude inertia (alone $\mathrm{t}-1$ ), and the interaction between alone $\mathrm{t}-1$ and depressive symptoms. The depression main effect was not associated with being alone $(\mathrm{OR}=1.00, \log \mathrm{OR}=0.00, \mathrm{SE}=0.02, \mathrm{p}=.828)$ indicating that depressive symptoms were not significantly associated with the probability of being alone in this sample. The solitude inertia effect, which captures how being alone at the previous experience sampling measure increases the probability of being alone, was positive and significant $(\mathrm{OR}=2.00, \log \mathrm{OR}=0.70, \mathrm{SE}=0.27, \mathrm{p}=.010)$. Participants were twice as likely to be alone when they were alone before. The estimate of the interaction between depressive symptoms and solitude inertia indicates that participants with one score more on the depression scale were $6 \%$ more likely to be alone when they were previously alone compared to those with one lower depression score $(\mathrm{OR}=1.06, \log \mathrm{OR}=0.06, \mathrm{SE}=0.03, \mathrm{p}=.012)$. Because odds ratios are not straightforward to interpret, we have visualized the predicted probability of being alone based on the (interaction) effects of previously being alone and depressive symptoms (see Figure 4). The solid line (incl. 95\% confidence band) shows the values that the model would predict of 'being alone' (Y-axis) based on individual's depression score (X-axis) and whether they were previously alone (blue = previously alone; red = previously in interaction). The (jittered) datapoints in Figure 4 additionally show the raw data and the dotted line show the fitted lines (incl. 95\% confidence band) based on the raw data. For example, based on the model a person with a depression score of 3 would have the probability of $\sim 40 \%$ to be alone after having previously been alone. A person with a depression score of 18 , on the other hand, would have a $\sim 60 \%$ probability of being along after having previously been alone. The size of the interaction effect becomes clearly visible in Figure 4; participants with higher depression scores are more likely to exhibit solitude inertia. The visualization also shows that the interaction effect is driven by individuals higher in depression being more likely to be alone after having been alone previously, while depression does not seem to change the probability of being alone if one was in an interaction before. Thus, participants with more depressive symptoms are equally likely to stay in a social interaction than participants low in depression. Hence, there is solitude inertia, but not social inertia associated with depression. 
Additionally, we have conducted numerous robustness analyses. The findings are robust in alternative model specifications (random intercept model, no covariates). Also, the same analysis was performed post hoc for the second experience sampling assessment period as a replication analysis $\left(\mathrm{T} 2, \mathrm{~N}_{\mathrm{obs}}=4,239\right)$. The results indicate similar dynamics, most importantly for the interaction between depression and previously being alone $(\mathrm{OR}=1.05, \log \mathrm{OR}=0.05$, $\mathrm{SE}=0.02, \mathrm{p}=.019)$. The results of these analyses can be found in the Supplementary Material Table 1. Furthermore, Table 2 in the Supplementary Material reports additional post hoc analyses with solitude inertia effects including not just $\mathrm{t}-1$, but $\mathrm{t}-2$ measures. Theses analyses indicate that the $\mathrm{t}-2$ being alone measure positively predicts being alone $(\mathrm{OR}=1.48, \log \mathrm{OR}=$ $0.39, \mathrm{p}=.028)$, but that this effect is not moderated by depressive symptoms at $\mathrm{T} 1(\mathrm{OR}=1.02$, $\log \mathrm{OR}=0.02, \mathrm{p}=.254$; model 1 of Table 2 in the Supplementary Material). However, when also considering the effects of being alone $\mathrm{t}-1$ on being alone (model 2 of Table 2 in the Supplementary Material), the solitude inertia effect of $\mathrm{t}-2$ disappears $(\mathrm{OR}=1.36, \log \mathrm{OR}=0.31$, $\mathrm{p}=.162)$. In these models, the depression related effects are all insignificant, besides the interaction with $\mathrm{t}-1$ solitude inertia $(\mathrm{OR}=1.05, \log \mathrm{OR}=0.45, \mathrm{p}=0.047)$.

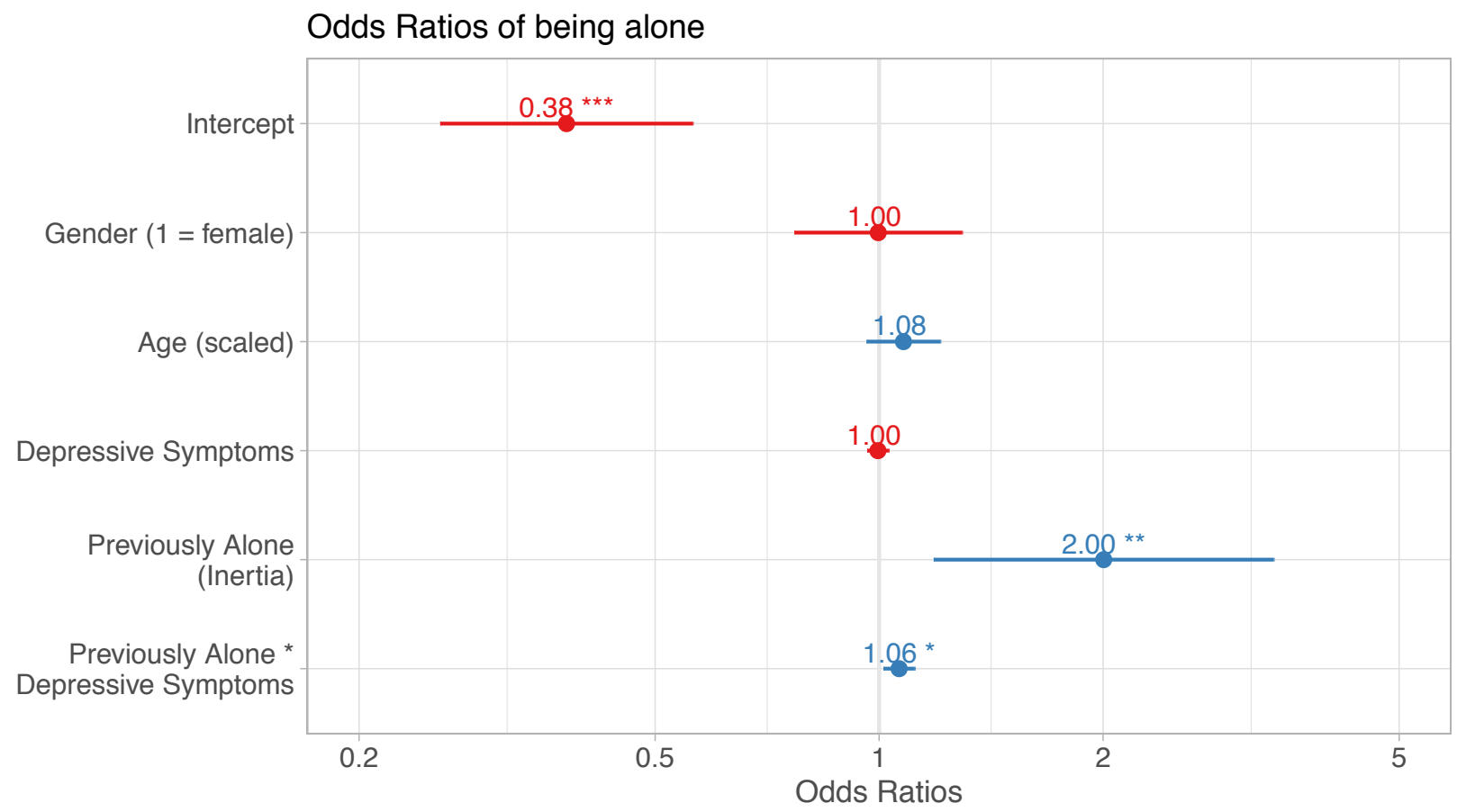

Figure 3. $N_{\text {obs }}=4,725, \mathrm{~N}_{\text {ind }}=129$. Odds Ratios of the logistic multilevel model on being alone during T1. $* * * \mathrm{p}<.001 ; * * \mathrm{p}<.01 ; * \mathrm{p}<.05$. A table of the detailed model results (incl. fit statistics) together with robustness analyses can be found in Supplementary Material Table 1. 


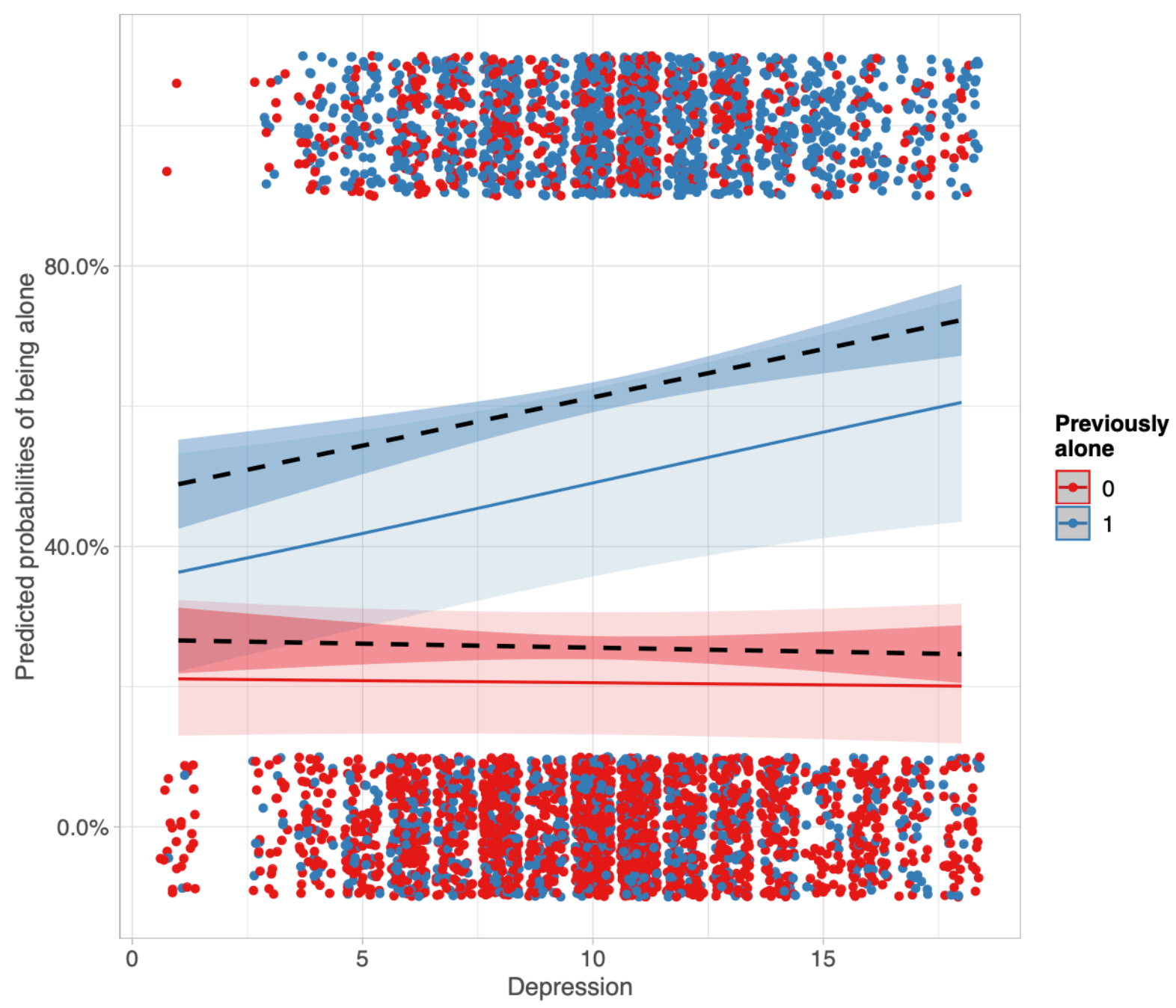

Figure 4. Predicted probabilities (solid line) and raw values (jittered data and dotted line) of being alone by depression and previously being alone. Confidence bands represent $95 \%$ confidence intervals. The predicted values are represented by the solid lines. The raw data is represented by points (with jitter) and the dotted black lines shows fitted lines based on the raw data. Blue points, lines, or error bars represent the values of previously being alone, whereas the color red represents not being alone previously.

\section{The effects of depressive symptoms on solitude inertia (RQ2)}

In this part, we test how depressive symptoms at T1 (assessment phase 1) predict solitude inertia at T2 (assessment phase 2) with a cross-lagged multilevel logistic regression model. Figure 5 shows the Odds Ratios of being alone at T2. The full model results are reported in Table 3 in the Supplementary Material. The single measure of depressive symptoms at T1 did not predict being alone at $\mathrm{T} 2(\mathrm{OR}=0.99, \log \mathrm{OR}=-0.01, \mathrm{SE}=0.02, \mathrm{p}=.800)$. The effect of the previous measurement of being alone on subsequently being alone (i.e., solitude inertia) was positive and significant $(\mathrm{OR}=2.60, \log \mathrm{OR}=0.97, \mathrm{SE}=0.31, \mathrm{p}=.002)$. The interaction effect 
of those two effects (i.e., previously being alone at T2 and the single depression measure at T1) was not significantly associated with being alone at $\mathrm{T} 2(\mathrm{OR}=1.04, \log \mathrm{OR}=0.04, \mathrm{SE}=0.03, \mathrm{p}$ $=.200$ ), indicating that individuals with more depressive symptoms at $\mathrm{T} 1 \mathrm{did}$ not exhibit more solitude inertia during T2.

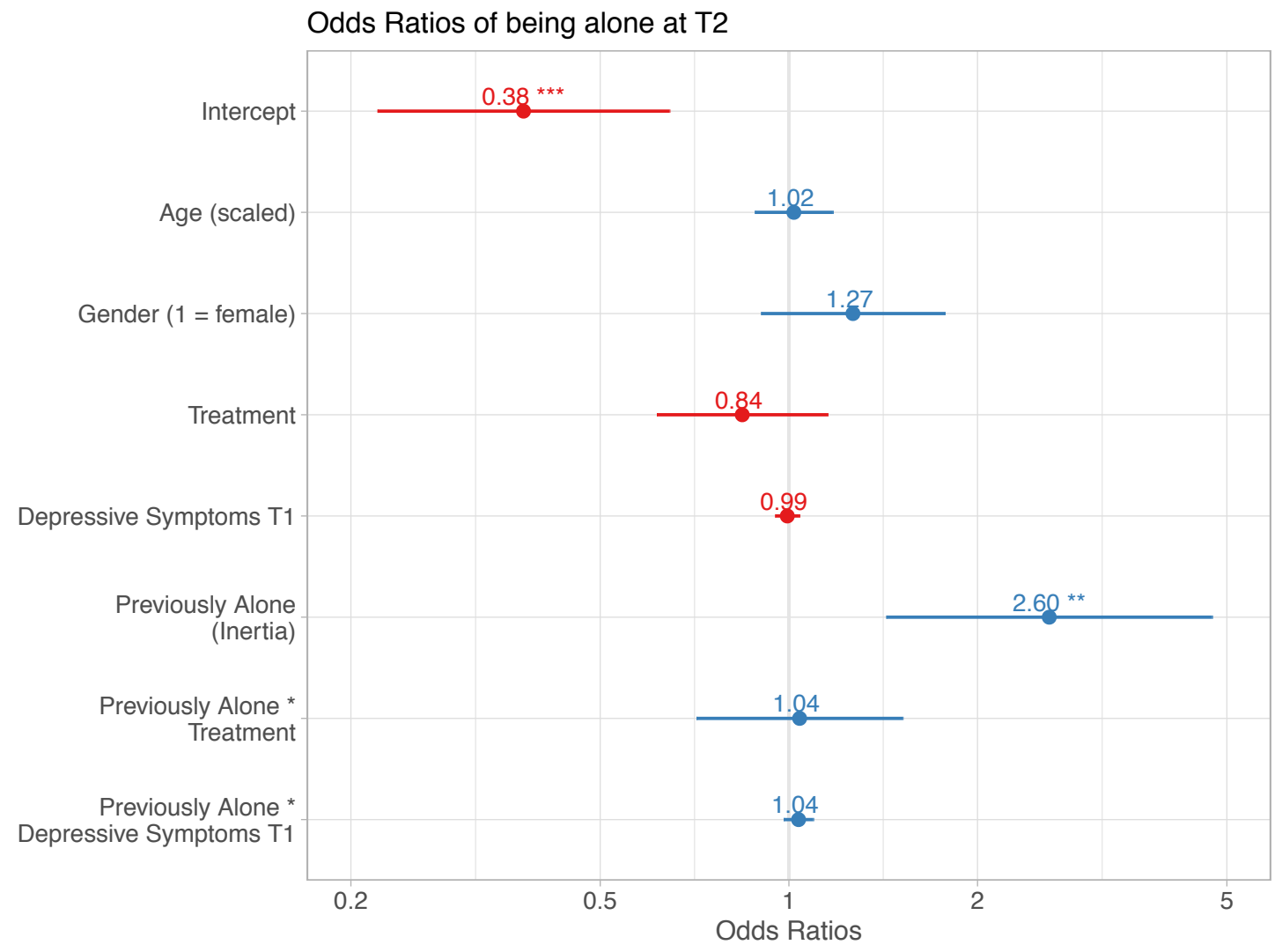

Figure 5. $\mathrm{N}_{\mathrm{obs}}=4,239, \mathrm{~N}_{\mathrm{ind}}=119$. Odds Ratios of the logistic multilevel model on being alone during T2. $* * * \mathrm{p}<.001 ; * * \mathrm{p}<.01 ; * \mathrm{p}<.05$. A table of the detailed model results (incl. fit statistics) together with robustness analyses can be found in the Supplementary Material Table 3.

\section{The effect of solitude inertia on depressive symptoms (RQ3)}

In this part, we test how solitude inertia at T1 predicts depression scores at T2. Figure 6 shows the estimates of a linear regression model predicting depressive symptoms T2. The full model results are reported in Table 4 of the Supplementary Material. Controlling for the previous depression measure (T1), the effect of treatment, age, gender, and the ratio of being alone in period 1, we find significant effects of the solitude inertia BLUP coefficients of T1 $(b=4.03, \mathrm{SE}$ $=1.89, \mathrm{p}=.035)$ on depressive symptoms in period two. This indicates that individuals who show higher solitude inertia (i.e., are more affected by previous measures of being alone on the 
current state of being alone) are more depressed eight weeks later. Moreover, we find no evidence for the effect of the ratio of being alone (social isolation) on depression eight weeks later $(b=-4.00, \mathrm{SE}=3.81, \mathrm{p}=.297)$. The model explained $25 \%$ of the variance.

We further ran an additional regression analysis, where the solitude inertia BLUP coefficients are taken from a multilevel model that does not have any Level 2 predictors (i.e., depression, age, gender). This way, the BLUP values represent solitude inertia irrespective of participants' depressive symptoms at T1. This robustness analysis is reported in Table 4 of the Supplementary Material and indicates that the raw BLUP values are significant predictors of depression at $\mathrm{T} 2$ as well $(\mathrm{b}=3.43, \mathrm{SE}=1.66, \mathrm{p}=.041)$. Furthermore, we report an additional analysis, where we control for the interaction between solitude inertia and the treatment condition. As shown in Model 3 of Table 4 in the Supplementary Material, the effect of the solitude inertia BLUP values was robust $(\mathrm{b}=4.16, \mathrm{SE}=2.09, \mathrm{p}=.049)$, and was not moderated by treatment condition $(b=-0.28, \mathrm{SE}=2.04, \mathrm{p}=.889)$.

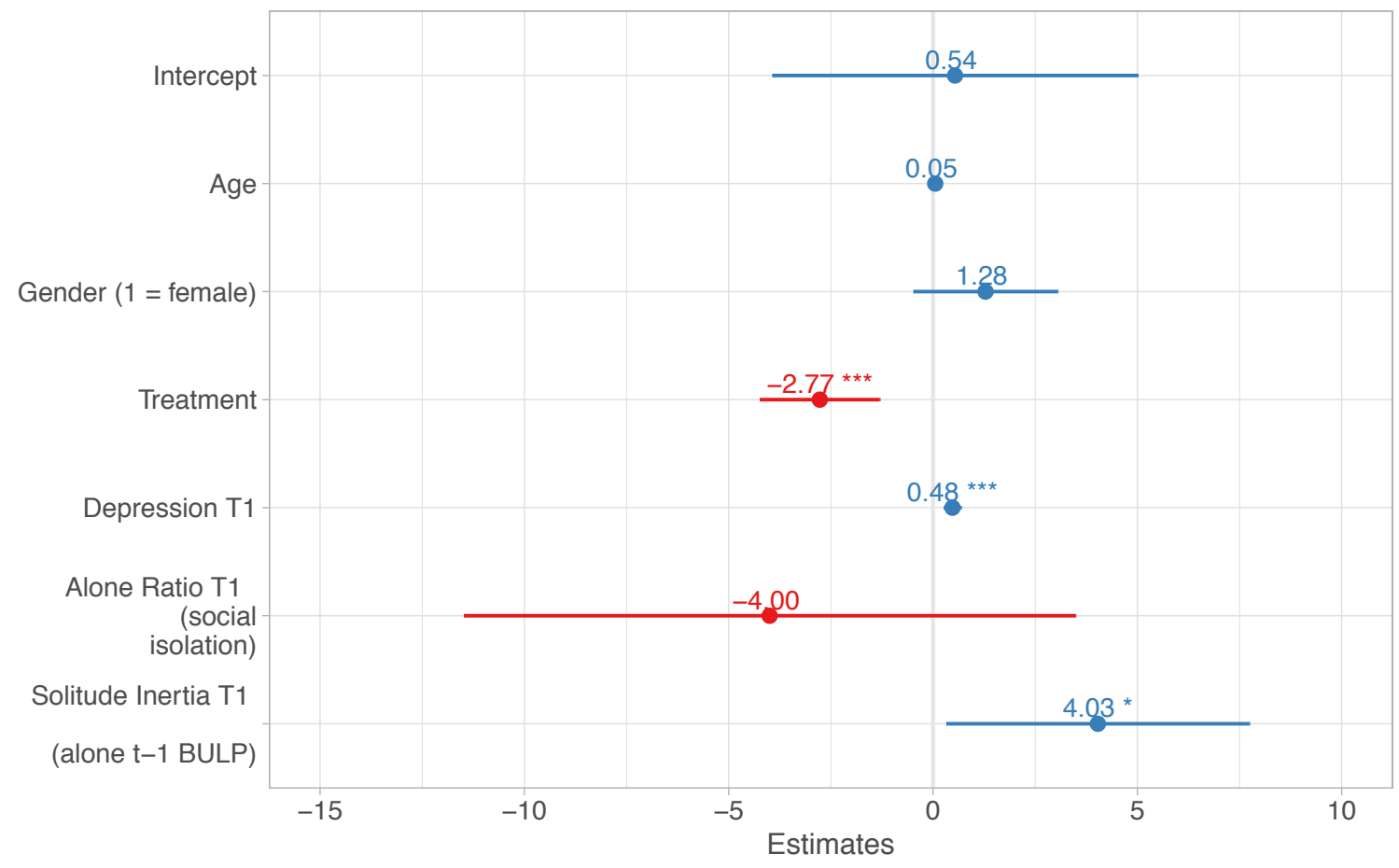

Figure 6. $\mathrm{N}=123 . \mathrm{R}^{2}=.25$. Linear Regression model results on depressive symptoms at $\mathrm{T} 2$. Error bars represent $95 \%$ confidence intervals. $* * * p<.001 ; * * p<.01 ; * p<.05$. 
As the solitude inertia BLUP values are not straightforward to interpret, in post hoc analyses we further computed the number of transitions between states of 'being alone' and 'not being alone'. For instance, a participant who was generally alone during one day but had one interaction during lunch would have two transitions (one from alone to the lunch interaction, and one from the interaction to alone). The number of transitions captures an alternative inverse measure of solitude inertia. We estimated another linear regression model with the number of transitions in T1 instead of the solitude inertia BLUP coefficients. The model results indicate that with each transition measured over the six-day T1 period, the depression score at T2 decreases by $0.25(\mathrm{SE}=0.07, \mathrm{p}<.001)$. Figure 7 shows the predicted values of depression at $\mathrm{T} 2$ by the number of transitions at T1. The figure indicates that individuals with more transitions between solitude and social interactions in $\mathrm{T} 1$ are less likely to be depressed at $\mathrm{T} 2$. The full model is reported as Model 5 in Supplementary Material Table 4. Additionally, we estimated a model with a normalized $\mathrm{T} 1$ transition predictor (i.e., number of transitions divided by the number of completed experience sampling measures). The results of this model, also indicate a negative effect of the normalized number of transitions during T1 on depressive symptoms at T2 $(\mathrm{b}=$ 14.97, $\mathrm{SE}=4.31, \mathrm{p}<.001)$. 


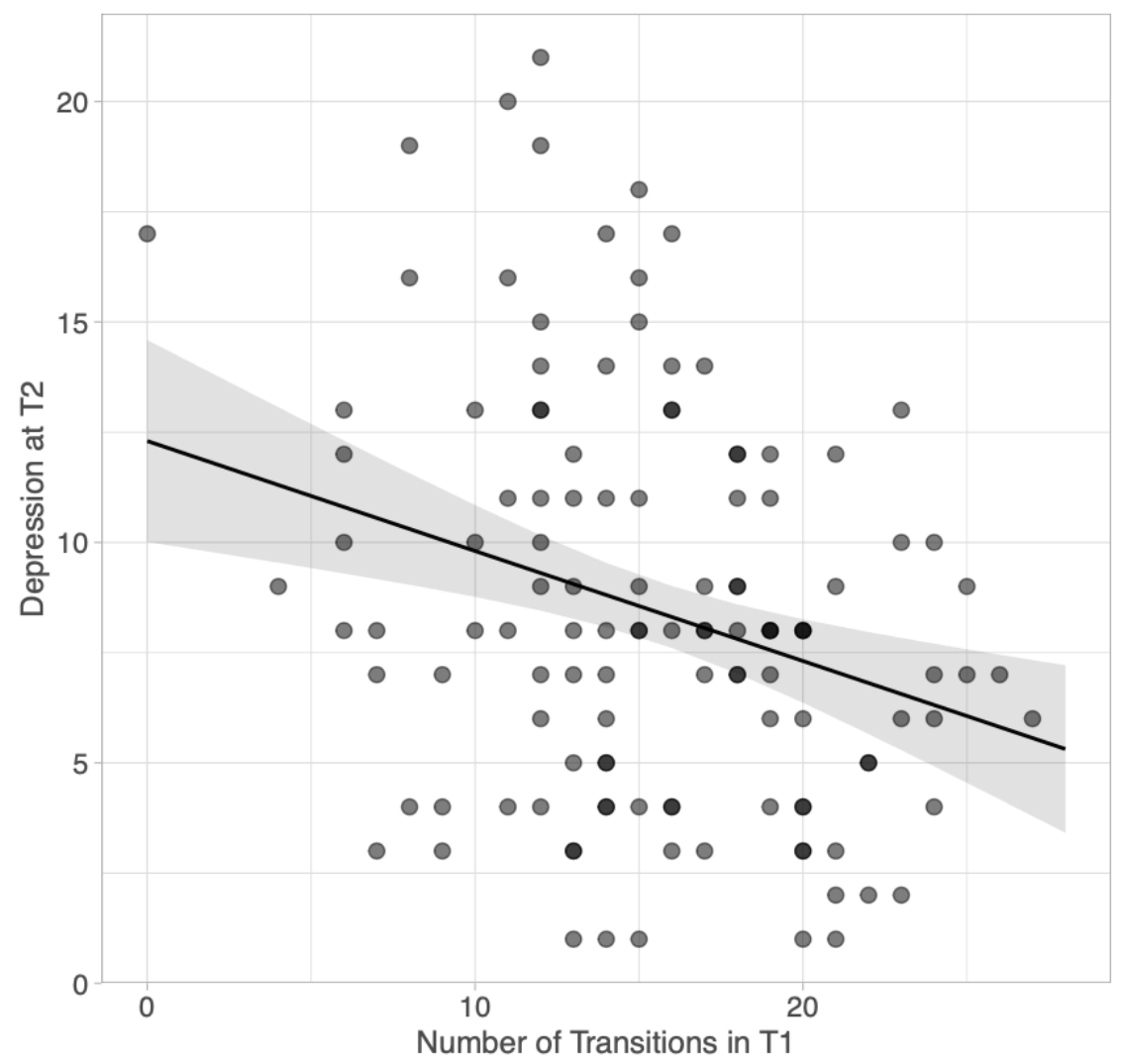

Figure 7. Predicted values (line with $95 \%$ confidence intervals) and raw values (points) of the number of transitions in $\mathrm{T} 1$ on the depression value of $\mathrm{T} 2$. 


\section{Discussion}

In this study, we investigated how solitude inertia - the tendency to remain in states of solitude and depressive symptoms are reinforcing each other in daily life. In that way, we examined the micro-level factors contributing to a vicious cycle between social isolation and depression.

Our analyses suggest that depressive symptoms are associated with exhibiting more solitude inertia. While the overall time individuals spend in social interactions is not associated with depressive symptoms, the (macro-level) depressive symptoms are statistically associated with the (micro-level) dynamics of 'being alone'. Moreover, the cross-lagged longitudinal analyses reveal that depressive symptoms at baseline do not predict solitude inertia eight weeks later. However, those that exhibit more solitude inertia at baseline are more likely to report more depressive symptoms eight weeks later.

These findings highlight the role of social interactions as important factors explaining the emergence and consequences of depression. Our analyses suggest that mixing patterns of solitude and social interactions manifest a healthy level of social self-regulation, as it is associated with a reduction of depressive symptoms in our data. This finding provides evidence for the long-term benefits of solitude phases between social interactions, in which one can reflect, process previous interactions, or reconnect to oneself (Coplan et al., 2017; Long \& Averill, 2003). So far, most existing studies solely focus on the short-term effects of social interaction dynamics on momentary emotional states (e.g., happiness, loneliness; Mote, Gonzalez, Kircos, Gard, \& Fulford, 2020; Quoidbach, Taquet, Desseilles, de Montjoye, \& Gross, 2019). For instance, Quoidbach and colleagues (2019) show that particular types of interactions (e.g., those with friends) are predictive of subsequent reports of happiness. Our study contributes to this line of research showing that general social interaction dynamics (solitude inertia) can have long-term effects on individuals' well-being and mental health.

We do not find evidence for a vicious cycle between solitude inertia and depression in this eight-week window between the two data-collection periods. The strength of the longitudinal design allowed us to disentangle the directionality of the positive association between concurrent measures of solitude inertia and depression. Our analyses suggest that this association is driven by the fact that solitude inertia is associated with an increase in depressive symptoms (and not 
vice versa). We thus contribute to the existing literature regarding reinforcing patterns between social factors and depressive symptoms (e.g., Cacioppo, Hawkley, \& Thisted, 2010; Cacioppo, Hughes, Waite, Hawkley, \& Thisted, 2006; Wichers, 2014; Wittenborn, Rahmandad, Rick, \& Hosseinichimeh, 2016) by investigating the directionality between a novel and dynamic social factor (solitude inertia) and depressive symptoms.

Future studies could, furthermore, test interventions that aim at disrupting solitude inertia by, for instance, training the individual's awareness about this mechanism and a following activation of social relationships. Mindfulness training that focuses on detecting and recognizing when solitude and social interaction is prolonged and negative, might serve as an important skill to foster. However, in our analyses the mindfulness treatment did not affect solitude inertia at post-measurement (for details see Supplementary Materials Table 3). Moreover, the role of individual's motivation to socially interact and the degree of derived pleasure (i.e., social anhedonia) could be investigated together with solitude inertia, as solitude inertia captures a dynamic and quantitative aspect of social interactions, but not their motives or qualities. Evidently, social intervention studies targeting solitude inertia would need to be evaluated empirically and put into perspective with existing subclinical interventions (e.g., befriending interventions; Siette, Cassidy, \& Priebe, 2017) or therapies (e.g., interpersonal psychotherapy; Klerman, Weissman, Rounsaville, \& Chevron, 1994).

This study has a number of limitations. First, we only investigated objective social isolation, thereby excluding the role that subjective social isolation (i.e., feeling lonely) plays in psychopathology (e.g., see Hawkley \& Cacioppo, 2010; van Roekel, Scholte, Engels, Goossens, \& Verhagen, 2015; van Winkel et al., 2017). People might exhibit solitude inertia but not necessarily feel lonely. How solitude inertia and trajectories of loneliness are related, could reveal more about the mechanisms behind objective and subjective social isolation. In that vein, it would also be interesting to relate solitude inertia to more fine-grained aspects of depressive symptoms, for instance, to the level of specific symptoms or to the level of momentary affect and their dynamics (Dejonckheere et al., 2019; Kuppens et al., 2012; van Winkel et al., 2017). Similarly, the quality of past social interactions, for instance, their pleasantness and closeness should also affect the patterns of future interactions and depression (Brown, Strauman, Barrantes-Vidal, Silvia, \& Kwapil, 2011; Nezlek, Hampton, \& Shean, 2000). 
Second, the experience sampling procedure, only samples social states of the participants. Thus, we do not know if between two measures of being alone the individual was always alone or whether interaction(s) occurred in between. On the other hand, even though our data suffers from this measurement error, we still find effects for the direction of solitude inertia to depression. The systematic trends that we found should only get more accurate and robust with a more reliable measure (Baugh, 2002). Nevertheless, future studies should investigate the continuum of social activities in daily life to capture solitude inertia in more detail. This can be achieved, for instance, with event-based diary studies (e.g., Ram et al., 2014), or automated interaction assessments (e.g., Elmer, Chaitanya, Purwar, \& Stadtfeld, 2019; Mehl, Pennebaker, Crow, Dabbs, \& Price, 2001).

\section{Conclusion}

Reinforcing patterns - whether vicious or beneficial - have been the interest of many psychological theories (Fredrickson \& Joiner, 2002; Wichers, 2014). This study highlights the role of solitude inertia, which represents a micro-level mechanism for the development of depressive symptoms. We find that solitude inertia is predictive of depressive symptoms eight weeks later, while there is no evidence for the predictive effects of depressive symptoms on solitude inertia. The fact that we did not find evidence for the presence of reinforcing patterns between solitude inertia and depressive symptoms further raises the question under which conditions such maladaptive reinforcing patters are apparent. Future studies on social interaction dynamics and solitude inertia should replicate the findings of this study and test intervention strategies reducing solitude inertia as one important explanatory mechanism to foster mental health and well-being. 


\section{References}

Atherton, B. D., Nevels, R. M., \& Moore, M. T. (2015). Predicting Symptoms of Depression From Social Anhedonia and Emotion Regulation. The Journal of Nervous and Mental Disease, 203(3), 170-174. http://doi.org/10.1097/NMD.0000000000000262

Baugh, F. (2002). Correcting effect sizes for score reliability: A reminder that measurement and substantive issues are linked inextricably. Educational and Psychological Measurement, 62(2), 254-263. http://doi.org/10.1177/0013164402062002004

Baumeister, R. F., \& Leary, M. R. (1995). The need to belong: Desire for interpersonal attachments as a fundamental human motivation. Psychological Bulletin, 117(3), 497-529. http://doi.org/10.1037/0033-2909.117.3.497

Bourdieu, P. (1985). The Social Space and the Genesis of Groups. Theory and Society, 14(6), 723-744. Retrieved from http://www.jstor.org/about/terms.html.

Brose, A., Schmiedek, F., Koval, P., \& Kuppens, P. (2015). Emotional inertia contributes to depressive symptoms beyond perseverative thinking. Cognition and Emotion, 29(3), 527538. http://doi.org/10.1080/02699931.2014.916252

Brown, L. H., Strauman, T., Barrantes-Vidal, N., Silvia, P. J., \& Kwapil, T. R. (2011). An experience-sampling study of depressive symptoms and their social context. The Journal of Nervous and Mental Disease, 199(6), 403-409. http://doi.org/10.1097/NMD.0b013e31821cd24b

Cacioppo, J. T., Hawkley, L. C., \& Thisted, R. A. (2010). Perceived social isolation makes me sad: 5-year cross-lagged analyses of loneliness and depressive symptomatology in the Chicago Health, Aging, and Social Relations Study. Psychology and Aging, 25(2), 453-463. http://doi.org/10.1037/a0017216

Cacioppo, J. T., Hughes, M. E., Waite, L. J., Hawkley, L. C., \& Thisted, R. A. (2006). Loneliness as a specific risk factor for depressive symptoms: Cross-sectional and longitudinal analyses. Psychology and Aging, 21(1), 140-151. http://doi.org/10.1037/08827974.21.1.140

Chappell, N. L., \& Badger, M. (1989). Social Isolation and Well-Being. Journal of Gerontology, 44(5), S169-S176. http://doi.org/10.1093/geronj/44.5.S169

Cohen, S., \& Ashby, T. (1985). Stress, Social Support, and the Buffering Hypothesis. Psychological Bulletin, 98(2), 310-57.

Coplan, R. J., Zelenski, J. M., \& Bowker, J. C. (2017). Leave Well Enough Alone? The Costs and Benefits of Solitude. In J. E. Maddux (Ed.), Subjective Well-Being and Life Satisfaction (pp. 130-143). New York, US: Routledge.

de Haan-Rietdijk, S., Voelkle, M. C., Keijsers, L., \& Hamaker, E. L. (2017). Discrete- vs. continuous-time modeling of unequally spaced experience sampling method data. Frontiers in Psychology, 8(OCT), 1-19. http://doi.org/10.3389/fpsyg.2017.01849

Dejonckheere, E., Mestdagh, M., Houben, M., Rutten, I., Sels, L., Kuppens, P., \& Tuerlinckx, F. (2019). Complex affect dynamics add limited information to the prediction of psychological well-being. Nature Human Behaviour, 3, 478-491. http://doi.org/10.1038/s41562-0190555-0

Elmer, T., Boda, Z., \& Stadtfeld, C. (2017). The co-evolution of emotional well-being with weak and strong friendship ties. Network Science, 5(3), 278-307. http://doi.org/10.1017/nws.2017.20

Elmer, T., Chaitanya, K., Purwar, P., \& Stadtfeld, C. (2019). The validity of RFID badges measuring face-to-face interactions. Behavior Research Methods, 1-19. 
http://doi.org/10.3758/s13428-018-1180-y

Evans, W. P., Marsh, S. C., \& Owens, P. (2007). Environmental factors, locus of control, and adolescent suicide risk. Child \& Adolescent Social Work Journal, 22(3-4), 301-319. http://doi.org/10.1007/bf02679474

Fredrickson, B. L., \& Joiner, T. (2002). Positive emotions trigger upward spirals toward emotional well-being. Psychological Science : A Journal of the American Psychological Society / APS, 13(2), 172-175. http://doi.org/10.1111/1467-9280.00431

Gable, S. L., \& Reis, H. T. (1999). Now and then, them and us, this and that: Studying relationships across time, partner, context, and person. Personal Relationships, 6, 415-432.

Geschwind, N., Peeters, F., Drukker, M., Van Os, J., \& Wichers, M. (2011). Mindfulness training increases momentary positive emotions and reward experience in adults vulnerable to depression: A randomized controlled trial. Journal of Consulting and Clinical Psychology, 79(5), 618-628. http://doi.org/10.1037/a0024595

Hamilton, M. (1960). A rating scale for depression. Journal of Neurology, Neurosurgery, and Psychiatry. http://doi.org/10.1136/jnnp.23.1.56

Hawkley, L. C., \& Cacioppo, J. T. (2010). Loneliness Matters: A Theoretical and Empirical Review of Consequences and Mechanisms. Annals of Behavioral Medicine, 40(2), 218-227. http://doi.org/10.1007/s12160-010-9210-8

Kawachi, I., \& Berkman, L. F. (2001). Social ties and mental health. Journal of Urban Health, 78(3), 458-467. http://doi.org/10.1093/jurban/78.3.458

Klerman, G. L., Weissman, M. M., Rounsaville, B. J., \& Chevron, E. S. (1994). Interpersonal Psychotherapy of Depression: A Brief, Focused, Specific Strategy. Oxford, UK: Rowman \& Litterfield. Retrieved from https://books.google.ch/books?hl=en\&lr=\&id=ZxuqnPWGcW8C\&oi=fnd\&pg=PP2\&dq=in fo:I8zZHKqi4MUJ:scholar.google.com\&ots=526969TPrR\&sig=ItMHLFGieEDfhkUYCRL XUTLz32M\&redir_esc $=\mathrm{y} \# \mathrm{v}=$ onepage $\& \mathrm{q} \& \mathrm{f}=$ false

Kuppens, P., Allen, N. B., \& Sheeber, L. B. (2010). Emotional inertia and psychological maladjustment. Psychological Science, 21(7), 984-991. http://doi.org/10.1177/0956797610372634

Kuppens, P., Sheeber, L. B., Yap, M. B. H., Whittle, S., Simmons, J. G., \& Allen, N. B. (2012). Emotional inertia prospectively predicts the onset of depressive disorder in adolescence. Emotion, 12(2), 283-289. http://doi.org/10.1037/a0025046

Kwapil, T. R., Silvia, P. J., \& Barrantes-Vidal, N. (2013). Social Anhedonia and Solitude. In R. J. Coplan \& J. C. Bowker (Eds.), The Handbook of Solitude. New York: Blackwell Publishing.

Larson, R. W. (1990). The solitary side of life: An examination of the time people spend alone from childhood to old age. Developmental Review, 10(2), 155-183. http://doi.org/10.1016/0273-2297(90)90008-R

Liu, S., Kuppens, P., \& Bringmann, L. (2019). On the Use of Empirical Bayes Estimates as Measures of Individual Traits. Assessment, 1073-1911. http://doi.org/10.1177/1073191119885019

Long, C. R., \& Averill, J. R. (2003). Solitude: An exploration of benefits of being alone. Journal for the Theory of Social Behaviour. http://doi.org/10.1111/1468-5914.00204

Mehl, M. R., Pennebaker, J. W., Crow, D. M., Dabbs, J., \& Price, J. H. (2001). The Electronically Activated Recorder (EAR): A device for sampling naturalistic daily activities and conversations. Behavior Research Methods, Instruments, \& Computers, 33(4), $517-$ 523. http://doi.org/10.3758/BF03195410

Mote, J., Gonzalez, R., Kircos, C., Gard, D., \& Fulford, D. (2020). I'm gonna make myself 
lonesome when you go: Predictors of loneliness in daily life. PsyArXiv. Retrieved from https://psyarxiv.com/pr79g/

Neubauer, A. B., Voelkle, M. C., Voss, A., \& Mertens, U. K. (2019). Estimating Reliability of Within-Person Couplings in a Multilevel Framework. Journal of Personality Assessment. http://doi.org/10.1080/00223891.2018.1521418

Nezlek, J. B., Hampton, C. P., \& Shean, G. D. (2000). Clinical depression and day-to-day social interaction in a community sample. Journal of Abnormal Psychology, 109(1), 11-9. http://doi.org/10.1037//0021-843X.109.1.11

Nolen-Hoeksema, S. (2000). The role of rumination in depressive disorders and mixed anxiety/depressive symptoms. Journal of Abnormal Psychology, 109(3), 504-11. Retrieved from http://www.ncbi.nlm.nih.gov/pubmed/11016119

Nolen-Hoeksema, S., \& Davis, C. G. (1999). "Thanks for sharing that": Ruminators and their social support networks. Journal of Personality and Social Psychology, 77(4), 801-814. http://doi.org/10.1037/0022-3514.77.4.801

Quoidbach, J., Taquet, M., Desseilles, M., de Montjoye, Y.-A., \& Gross, J. J. (2019). Happiness and Social Behavior. Psychological Science. http://doi.org/10.1177/0956797619849666

Ram, N., Conroy, D. E., Pincus, A. L., Lorek, A., Rebar, A., Roche, M. J., ... Gerstorf, D. (2014). Examining the Interplay of Processes Across Multiple Time-Scales: Illustration With the Intraindividual Study of Affect, Health, and Interpersonal Behavior (iSAHIB). Research in Human Development, 11(2), 142-160. http://doi.org/10.1080/15427609.2014.906739

Rottenberg, J., \& Gotlib, I. H. (2008). Socioemotional Functioning in Depression. In M. Power (Ed.), Mood Disorders: A Handbook of Science and Practice. New York: John Wiley \& Sons Inc.

Santini, Z., Koyanagi, A., Tyrovolas, S., Mason, C., \& Maria, J. (2015). The association between social relationships and depression: A systematic review. Journal of Affective Disorders, 175, 53-65. http://doi.org/10.1016/j.jad.2014.12.049

Siette, J., Cassidy, M., \& Priebe, S. (2017, April 1). Effectiveness of befriending interventions: A systematic review and meta-analysis. BMJ Open. BMJ Publishing Group. http://doi.org/10.1136/bmjopen-2016-014304

Silvia, P. J., \& Kwapil, T. R. (2011). Aberrant Asociality: How Individual Differences in Social Anhedonia Illuminate the Need to Belong. Journal of Personality, 79(6), 1315-1332. http://doi.org/10.1111/j.1467-6494.2010.00702.x

Suls, J., Green, P., \& Hillis, S. (1998). Emotional reactivity to everyday problems, affective inertia, and neuroticism. Personality and Social Psychology Bulletin, 24(2), 127-136. http://doi.org/10.1177/0146167298242002

Teasdale, J. D., Segal, Z. V, Mark, J., Williams, G., Ridgeway, V. A., Soulsby, J. M., \& Lau, M. A. (2000). Prevention of Relapse/Recurrence in Major Depression by Mindfulness-Based Cognitive Therapy. Journal of Consulting and Clinical Psychology Psychological Association, Inc, 68(4), 615-623. http://doi.org/10.1037/0022-006X.68.4.615

van Roekel, E., Scholte, R. H. J., Engels, R. C. M. E., Goossens, L., \& Verhagen, M. (2015). Loneliness in the Daily Lives of Adolescents: An Experience Sampling Study Examining the Effects of Social Contexts. Journal of Early Adolescence, 35(7), 905-930. http://doi.org/10.1177/0272431614547049

van Winkel, M., Wichers, M., Collip, D., Jacobs, N., Derom, C., Thiery, E., ... Peeters, F. (2017). Unraveling the Role of Loneliness in Depression: The Relationship Between Daily Life Experience and Behavior. Psychiatry (New York), 80(2), 104-117. http://doi.org/10.1080/00332747.2016.1256143 
Wichers, M. (2014). The dynamic nature of depression: a new micro-level perspective of mental disorder that meets current challenges. Psychological Medicine, 616(March), 1-12. http://doi.org/10.1017/S0033291713001979

Wittenborn, A. K., Rahmandad, H., Rick, J., \& Hosseinichimeh, N. (2016). Depression as a systemic syndrome: Mapping the feedback loops of major depressive disorder. Psychological Medicine, 46(3), 551-562. http://doi.org/10.1017/S0033291715002044 